\title{
Prevalence and incidence rate of diabetes mellitus in a Swedish community during 30 years of follow-up
}

\author{
S. P. O. Jansson • D. K. G. Andersson • K. Svärdsudd
}

Received: 10 July 2006 / Accepted: 5 December 2006 / Published online: 1 February 2007

(C) Springer-Verlag 2007

\begin{abstract}
Aims/hypothesis Increasing diabetes prevalence has been reported in most European countries in the last 20 years. In this study we report on the development of prevalence and incidence of diabetes from 1972 to 2001 in Laxå, a rural community in central Sweden.

Materials and methods A diabetes register was established at the primary healthcare centre (PHCC) in Laxå, beginning in 1972 and based on data from clinical records at the PHCC, nearby hospitals and private practitioners in the area. In addition, case-finding procedures involving $85 \%$ of the residents aged 35 to 79 years old was performed from 1983 onwards.

Results During the study period a total of 776 new diabetes cases was found, 36 type 1 diabetes mellitus and 740 type 2 diabetes mellitus. The age-standardised incidence rates for type 1 diabetes mellitus and type 2 diabetes mellitus were 0.15 and 3.03 cases per 1,000 population, respectively. No increase in incidence over time was detected for either forms of diabetes. Age-standardised prevalence for women and men increased from 28.3 and 25.9, respectively, per 1,000 in 1972 to 45 and 46.3 per 1,000 in $1988(p<0.0001)$, thereafter falling to a mean of 43.5 per 1,000 for women, while men had a mean of 44.9 per 1,000 for the rest of the study period.
\end{abstract}

\section{S. P. O. Jansson $(\bowtie)$}

Department of Clinical Medicine, Family Medicine Research Centre, Örebro University,

Box 1613, 70116 Örebro, Sweden

e-mail: stefan.jansson@orebroll.se

S. P. O. Jansson • D. K. G. Andersson · K. Svärdsudd Department of Public Health and Caring Sciences, Family Medicine and Clinical Epidemiology Section, Uppsala University, Uppsala, Sweden
Conclusions/interpretation The prevalence of diabetes mellitus in Laxå is high, but has not increased during the last 13 years. The incidence rate was relatively stable over the whole 30-year period.

Keywords Case-finding · Diabetes mellitus · Incidence · Prevalence . Time trend

\section{Abbreviation \\ PHCC primary healthcare centre}

\section{Introduction}

Diabetes is one of the main contributors to ill health and premature mortality worldwide [1]. The global prevalence for all age groups is expected to increase from $2.8 \%$ in 2000 to $4.4 \%$ in 2030 . The corresponding number of subjects with diabetes would then increase from 171 million to 366 million [2]. Possible causes of this 'diabetes epidemic' are: population growth, longer survival, urbanisation, low physical activity, obesity, excessive energy intake and other factors.

However, the existence of an epidemic has been debated [3]. In recent decades, a number of prevalence rates have been reported from population samples of various ages [4-7]. A few studies have reported on prevalence rate time trends. In Denmark, the prevalence of diabetes among 60-year-old subjects increased from $7.8 \%$ in 1974 to $12.3 \%$ in 1996 among men and from 5.6 to $6.8 \%$ among women [8]. In a Norwegian study based on surveys in 1984 and 1995 of all inhabitants over the age of 20 , the diabetes prevalence increased from 2.9 to $3.2 \%$, but only in men [9]. In northern Sweden, no increase in prevalence was found between 1986 
and 1999 on the basis of four population surveys of 25- to 64-year-old subjects [10]. In contrast, on the basis of a Swedish national interview survey of subjects aged 16 to 84 years, an increase in prevalence of $1.1 \%$ among men and $0.7 \%$ among women was found for the periods $1980-1987$ to 1996-2003 [11].

A problem inherent in this type of study design is whether the observed change is a true one or whether it has to do with structural changes over time in the study base, such as ageing population, or secular or generational effects. To overcome these problems, we performed a diabetes prevalence study for the period between 1972 and 1989 in a community where all new diabetes cases were systematically registered [4]. In this report, prevalence and incidence rates for the time period 1972 to 2001 are presented.

\section{Subjects and methods}

Setting The study was undertaken in Laxå, a small municipality of $565 \mathrm{~km}^{2}$ in Örebro county, central Sweden. The study base consisted of the Laxå municipality population during the period 1972 to 2001. The Laxå population decreased and grew older over time. This trend was stronger than that for Örebro county and the Swedish national population. The distribution of professions, unemployment rate, average income and urban/rural areas was approximately the same as in the national population.

Healthcare in Laxå is provided by the local primary healthcare centre (PHCC), which is part of the Örebro County Health Service. One private practitioner worked part-time outside the PHCC from 1980 to 1997 . The nearest hospitals, Karlskoga and Örebro, are located 45 and $52 \mathrm{~km}$ away. The vast majority of Laxå residents with type 2 diabetes were treated at the PHCC and most subjects with type 1 diabetes were treated at one of the two hospitals.

Diabetes registry From 1 January 1972 onwards all prevalent and incident diabetes patients in Laxå were entered in a diabetes register at Laxå PHCC. To identify possible missing cases, patient record files at nearby PHCCs, private practitioners and hospitals (including Departments of Ophthalmology) were checked. In addition, prescriptions for insulin, metformin, sulfonylureas, syringes and urine/blood glucose test material filed at the local pharmacy were scrutinised for 6 months in 1987 and for 9 months in 1991. However, no additional, previously unknown diabetic patients were found using these procedures.

Identification of new diabetes cases In the clinical setting, patients were liberally tested for random capillary wholeblood glucose, especially if they had a family history of diabetes, as well as for impaired glucose tolerance or potential diabetes co-morbidities such as hypertension, ischaemic heart disease, obesity or dyslipidaemia.

Along with the clinical activities, case-finding procedures were performed in 5-year cycles as follows: 19831987, 1988-1992, 1993-1997 and 1998-2001 (4 years only due to study end). All individuals aged 35 to 79 years who visited the PHCC for whatever reason and who agreed to participate underwent, at least once during a 5-year period, random capillary whole-blood glucose and urine glucose tests. Subjects with a high risk of developing diabetes were offered blood glucose tests yearly. High-risk subjects were defined as those with previously known hypertension, cerebro-cardiovascular disease, dyslipidaemia, first-degree heredity for diabetes and those who were overweight or taking medications such as corticosteroids or immunosuppressive drugs. Approximately $85 \%$ of the eligible Laxå population was screened. Screening tests for blood glucose were considered to be positive at the following values: $\geq 8.0 \mathrm{mmol} / 1$ for $1983-1987$ [12], $\geq 7.5 \mathrm{mmol} / 1$ for $1988-1992$ and 1993-1997, and $\geq 6.7 \mathrm{mmol} / \mathrm{l}$ for $1998-2001$.

For those who screened positive, a diagnostic work-up procedure was performed including fasting blood glucose measurements on at least two separate occasions. If these were in the range 5.5 to 6.9 (WHO 1980 criteria) or 4.4 to $6.6 \mathrm{mmol} / \mathrm{l}$ (WHO 1985 criteria), a 75-g OGTT was performed at the PHCC and interpreted according to the 1980, 1985 and 1999 WHO criteria [13-15].

For all cases detected, the WHO diabetes diagnosis criteria from 1980, 1985 and 1999 [13-15] were used during the appropriate periods. Diabetes was classified as type 1 in: (1) patients with onset before age 30 years and needing insulin treatment within one year of diagnosis; (2) patients with disease onset after age 30 years when prompt insulin treatment was necessary; or (3) patients with slowly developing insulin-dependence, plus islet antibodies or very low C-peptide secretion on glucagon stimulation. Type 2 diabetes was diagnosed when patients had: (1) disease onset at age $\geq 30$ years with no ketonuria at diagnosis and no prompt need for insulin treatment; or (2) disease onset $<30$, but were well controlled using diet or oral hypoglycaemic agents for several years, with no islet antibodies. Date of first diagnosis was regarded as time of onset.

Data on the number of Laxå residents, classified by sex and 5-year age groups on 31 December for each of the study years, and the corresponding data for the national Swedish population in 1987 were obtained from Statistics Sweden. Data on migration in and out of the area were obtained from the population register. Mortality data were obtained from the National Cause of Death Registry. The study was approved by the Örebro Research Ethics Committee. 
Statistical analysis The data were analysed using the SAS software package, release 6.12 and JMP software package, release 5.0.1.2 (SAS Institute, Cary, NC, USA). Incident cases were defined as new cases diagnosed in Laxå in a specific year and still living in Laxå on 31 December of that year. Prevalent cases for a specific year were defined as cases with diagnosed diabetes living in Laxå on 31 December of that year. The number of incident and prevalent cases was age-standardised in 5-year age intervals against the Swedish national population in 1987. Prevalence was calculated as number of age-standardised prevalent cases per head of population on 31 December of each of the study years, accounting for migration into and out of Laxå and survival. The incidence rate was calculated as the number of age-standardised new cases diagnosed in Laxå per head of population on 31 December of the diagnosis year. A data set including all incident and prevalent cases and all non-cases in Laxå for each calendar year was created. Trends for prevalence and incidence rates across calendar time were tested in that dataset using logistic regression with diabetes status (yes/no) as dependent variable and calendar year (and its square to account for curvilinear relationships) as independent variable; this was done separately for men and women and adjusted for age. We regarded $p$ values of less than $5 \%$ as statistically significant.

\section{Results}

During the study period 1972-2001, a total of 776 new cases of diabetes with a male:female ratio of 1.07 was found (Table 1). Of these, 36 cases were type 1 diabetes mellitus and 740 type 2 diabetes mellitus. The total age-

Table 1 Incidence of diabetes expressed as age-standardised number of new cases per 1,000 residents each year in the community of Laxå

\begin{tabular}{|c|c|c|c|c|c|c|c|c|}
\hline \multirow[b]{3}{*}{ Year } & \multicolumn{4}{|c|}{ Crude number of new cases } & \multicolumn{4}{|c|}{ Age-standardised number of new cases per 1,000 residents } \\
\hline & \multicolumn{2}{|l|}{ Type 1} & \multicolumn{2}{|l|}{ Type 2} & \multicolumn{2}{|l|}{ Type 1} & \multicolumn{2}{|l|}{ Type 2} \\
\hline & Women & Men & Women & Men & Women & Men & Women & Men \\
\hline 1972 & 0 & 0 & 16 & 12 & 0.0 & 0.0 & 4.4 & 2.7 \\
\hline 1973 & 3 & 1 & 8 & 9 & 0.6 & 0.2 & 2.3 & 2.1 \\
\hline 1974 & 0 & 0 & 13 & 17 & 0.0 & 0.0 & 3.8 & 3.8 \\
\hline 1975 & 2 & 1 & 13 & 11 & 0.5 & 0.2 & 3.9 & 2.3 \\
\hline 1976 & 1 & 0 & 12 & 9 & 0.2 & 0.0 & 3.2 & 1.8 \\
\hline 1977 & 0 & 0 & 13 & 22 & 0.0 & 0.0 & 3.5 & 4.8 \\
\hline 1978 & 1 & 0 & 9 & 12 & 0.3 & 0.0 & 2.5 & 2.7 \\
\hline 1979 & 0 & 1 & 12 & 12 & 0.0 & 0.2 & 3.0 & 2.6 \\
\hline 1980 & 0 & 0 & 15 & 17 & 0.0 & 0.0 & 3.7 & 3.8 \\
\hline 1981 & 0 & 0 & 12 & 10 & 0.0 & 0.0 & 3.0 & 2.2 \\
\hline 1982 & 0 & 3 & 8 & 13 & 0.0 & 0.7 & 2.0 & 2.8 \\
\hline 1983 & 0 & 1 & 19 & 19 & 0.0 & 0.2 & 4.3 & 4.2 \\
\hline 1984 & 0 & 0 & 11 & 15 & 0.0 & 0.0 & 2.7 & 3.2 \\
\hline 1985 & 2 & 1 & 21 & 17 & 0.4 & 0.2 & 5.2 & 3.9 \\
\hline 1986 & 1 & 1 & 12 & 16 & 0.3 & 0.2 & 3.0 & 3.5 \\
\hline 1987 & 1 & 0 & 18 & 14 & 0.2 & 0.0 & 4.6 & 3.4 \\
\hline 1988 & 1 & 2 & 12 & 22 & 0.3 & 0.5 & 2.9 & 5.2 \\
\hline 1989 & 1 & 0 & 10 & 7 & 0.3 & 0.0 & 2.6 & 1.5 \\
\hline 1990 & 0 & 2 & 12 & 10 & 0.0 & 0.5 & 3.0 & 2.3 \\
\hline 1991 & 0 & 1 & 11 & 10 & 0.0 & 0.3 & 2.8 & 2.4 \\
\hline 1992 & 1 & 1 & 5 & 9 & 0.3 & 0.3 & 1.4 & 2.4 \\
\hline 1993 & 1 & 0 & 9 & 6 & 0.3 & 0.0 & 2.4 & 1.4 \\
\hline 1994 & 0 & 0 & 10 & 14 & 0.0 & 0.0 & 2.8 & 3.4 \\
\hline 1995 & 0 & 0 & 7 & 9 & 0.0 & 0.0 & 1.9 & 2.3 \\
\hline 1996 & 0 & 0 & 16 & 15 & 0.0 & 0.0 & 4.2 & 3.9 \\
\hline 1997 & 0 & 1 & 14 & 14 & 0.0 & 0.4 & 3.6 & 3.2 \\
\hline 1998 & 0 & 0 & 9 & 4 & 0.0 & 0.0 & 2.4 & 0.8 \\
\hline 1999 & 0 & 2 & 6 & 9 & 0.0 & 0.6 & 1.9 & 2.5 \\
\hline 2000 & 0 & 0 & 13 & 14 & 0.0 & 0.0 & 3.5 & 3.7 \\
\hline 2001 & 2 & 1 & 12 & 14 & 0.7 & 0.3 & 3.0 & 3.6 \\
\hline Total & 17 & 19 & 358 & 382 & 0.15 & 0.16 & 3.11 & 2.95 \\
\hline
\end{tabular}


Fig. 1 Incidence of diabetes for women expressed as agestandardised number of new cases per 1,000 residents per year in the community of Laxå. Blue line, women 0-24 years; red line, women 2534 years; yellow line, women 35-44 years; green line, women 45-54 years; purple line, women 55-64 years; orange line, women 65-74 years; brown line, women 75 years and older; black line, all women

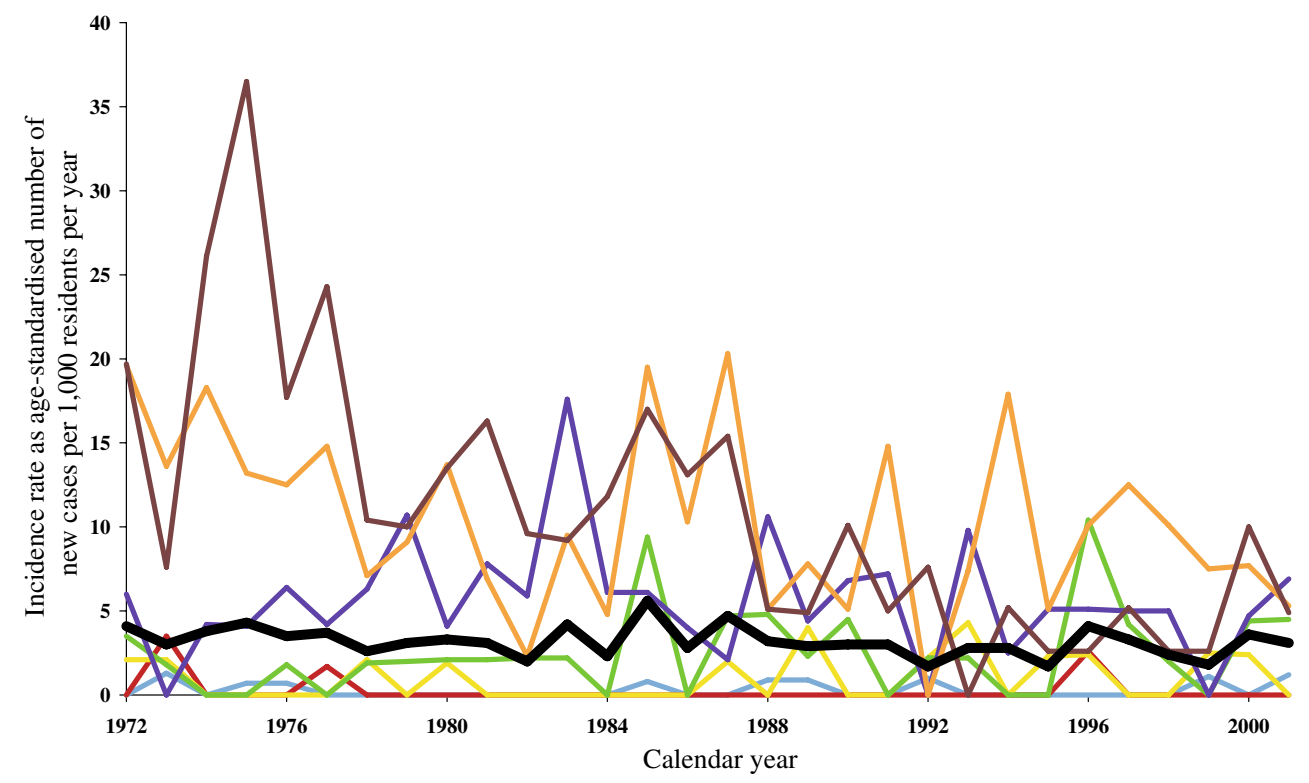

standardised incidence rate for type 1 diabetes mellitus was 0.15 per 1,000 population and for type 2 diabetes mellitus 3.03 per 1,000 population. For all age classes the incidence rates, for women as well as for men, varied yearly (Figs. 1 and 2), with the total age-standardised incidence rates for women and men fluctuating between a high of 5.6 and 5.5 cases per 1,000 population, respectively, in the period 1985-1988 (which also represented the first case-finding period) and a low of 1.7 and 0.9 cases per 1,000 population, respectively, for the period 1992-1998 (a non-significant change) (Table 1, Figs. 1 and 2). There was no increase in total incidence for all diabetes over time.

The crude type 1 and type 2 diabetes mellitus prevalence increased over time for women and for men (not shown) through the study period. Age-standardised prevalence for women and men increased from 28.3 and 25.9 per 1,000 , respectively, in 1972 to 45 and 46.3 per 1,000, respectively, in $1988(p<0.0001)$, thereafter falling for the rest of the study period to a mean of 43.5 per 1,000 for women, while men had a mean of 44.9 per 1,000 (Table 2, Figs. 3 and 4). The male:female ratio varied over time. For women aged 0-44 years the prevalence was fairly constant through the study period, whereas for women in the 45-54 and 5564 year age groups, the prevalence increased until 1988 and 1993, respectively, subsequently decreasing slightly. The prevalence for women aged 65-74 years increased until 1987 and then fell (Fig. 3). For men aged 0-24 years the prevalence was constantly low throughout the study period, while for men aged 25-34 years the prevalence increased until 1985 and then remained stable for the rest of the
Fig. 2 Incidence of diabetes for men expressed as agestandardised number of new cases per 1,000 residents per year in the community of Laxå Blue line, men 0-24 years; red line, men 25-34 years; yellow line, men 35-44 years; green line, men 45-54 years; purple line, men 55-64 years; orange line, men 65 74 years; brown line, men 75 years and older; black line, all men

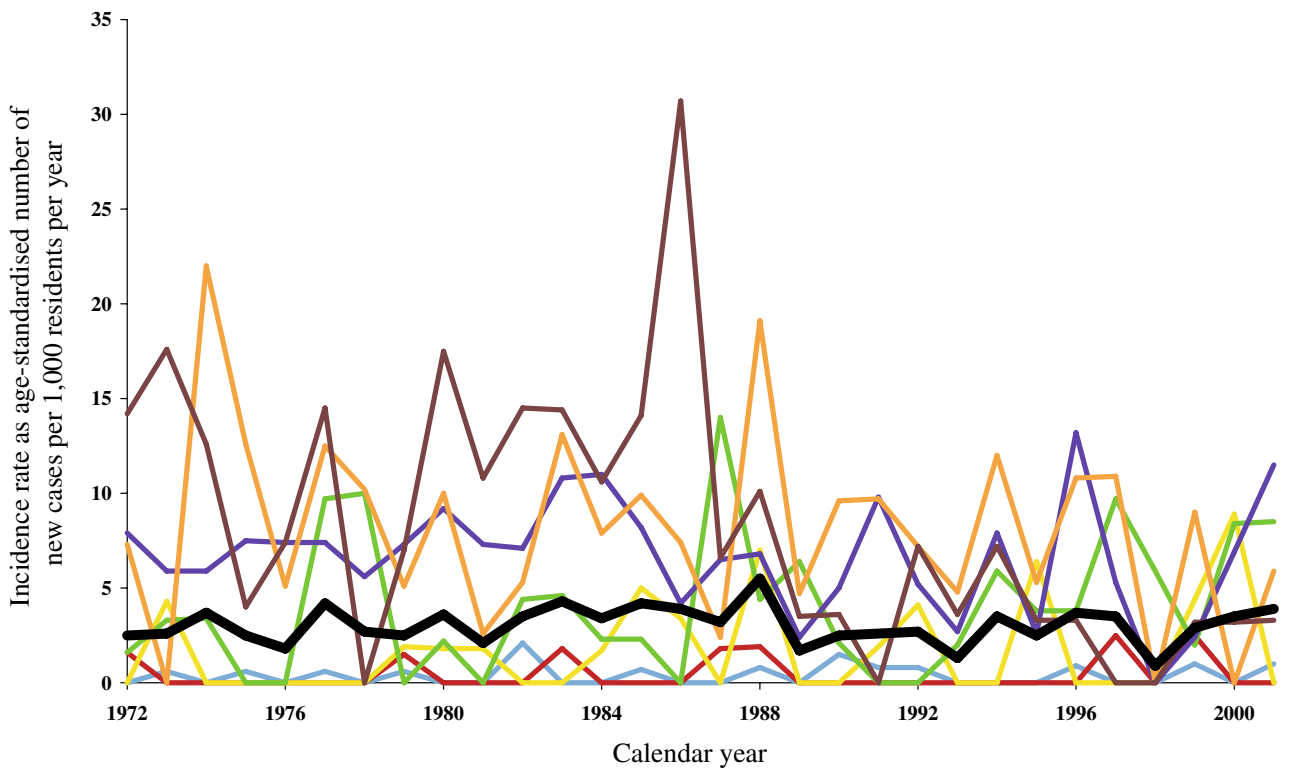


Table 2 Prevalence of diabetes expressed as age-standardised number of prevalent cases per 1,000 residents each year in the community of Laxå

\begin{tabular}{|c|c|c|c|c|c|c|c|c|}
\hline \multirow[b]{3}{*}{ Year } & \multicolumn{4}{|c|}{ Crude number of prevalent cases } & \multicolumn{4}{|c|}{ Age-standardised number of prevalent cases per 1,000 residents } \\
\hline & \multicolumn{2}{|l|}{ Type 1} & \multicolumn{2}{|l|}{ Type 2} & \multicolumn{2}{|l|}{ Type 1} & \multicolumn{2}{|l|}{ Type 2} \\
\hline & Women & Men & Women & Men & Women & Men & Women & Men \\
\hline 1972 & 11 & 19 & 89 & 92 & 2.7 & 4.2 & 25.6 & 21.7 \\
\hline 1973 & 14 & 20 & 91 & 94 & 3.0 & 4.8 & 26.1 & 22.0 \\
\hline 1974 & 14 & 19 & 100 & 102 & 3.1 & 4.4 & 28.5 & 23.1 \\
\hline 1975 & 15 & 20 & 108 & 107 & 3.6 & 4.5 & 31.3 & 23.9 \\
\hline 1976 & 16 & 20 & 115 & 109 & 3.7 & 4.6 & 32.9 & 23.9 \\
\hline 1977 & 15 & 18 & 122 & 121 & 3.3 & 4.2 & 34.7 & 26.8 \\
\hline 1978 & 16 & 18 & 123 & 126 & 3.7 & 4.2 & 34.7 & 28.5 \\
\hline 1979 & 16 & 18 & 125 & 127 & 4.0 & 4.0 & 34.1 & 27.5 \\
\hline 1980 & 14 & 17 & 136 & 138 & 3.5 & 3.8 & 36.4 & 29.3 \\
\hline 1981 & 14 & 16 & 140 & 132 & 3.6 & 3.7 & 36.7 & 28.8 \\
\hline 1982 & 14 & 19 & 136 & 136 & 3.7 & 4.5 & 35.7 & 29.5 \\
\hline 1983 & 14 & 21 & 143 & 151 & 3.7 & 4.8 & 37.1 & 32.9 \\
\hline 1984 & 13 & 20 & 141 & 155 & 3.3 & 4.8 & 36.3 & 33.8 \\
\hline 1985 & 15 & 21 & 152 & 160 & 3.9 & 5.1 & 38.3 & 35.3 \\
\hline 1986 & 14 & 22 & 155 & 168 & 3.6 & 5.7 & 38.4 & 37.0 \\
\hline 1987 & 14 & 23 & 165 & 169 & 3.6 & 5.7 & 40.9 & 37.3 \\
\hline 1988 & 13 & 24 & 166 & 182 & 3.4 & 6.0 & 41.6 & 40.3 \\
\hline 1989 & 15 & 24 & 165 & 175 & 4.0 & 6.2 & 40.4 & 38.4 \\
\hline 1990 & 13 & 25 & 167 & 164 & 3.5 & 6.9 & 41.5 & 36.9 \\
\hline 1991 & 12 & 25 & 165 & 159 & 3.3 & 7.0 & 41.3 & 36.6 \\
\hline 1992 & 13 & 26 & 161 & 157 & 3.9 & 7.4 & 40.5 & 36.9 \\
\hline 1993 & 14 & 26 & 158 & 157 & 4.2 & 7.7 & 40.2 & 36.6 \\
\hline 1994 & 14 & 25 & 158 & 165 & 4.0 & 7.5 & 41.0 & 37.9 \\
\hline 1995 & 13 & 24 & 153 & 161 & 3.8 & 7.2 & 39.5 & 37.5 \\
\hline 1996 & 11 & 23 & 158 & 164 & 3.2 & 7.1 & 40.0 & 39.0 \\
\hline 1997 & 11 & 24 & 152 & 168 & 3.3 & 7.5 & 39.1 & 39.8 \\
\hline 1998 & 9 & 21 & 152 & 163 & 3.0 & 7.2 & 38.9 & 37.9 \\
\hline 1999 & 8 & 24 & 151 & 159 & 2.4 & 8.1 & 38.2 & 36.3 \\
\hline 2000 & 8 & 22 & 159 & 160 & 2.4 & 7.1 & 40.6 & 37.7 \\
\hline 2001 & 10 & 24 & 159 & 162 & 3.7 & 7.8 & 40.7 & 39.3 \\
\hline
\end{tabular}

period. For men in the 35-44 year age group the prevalence increased from 1984 to 1995 and then fell. The prevalence for men aged 45-64 years increased over time, as it did for men aged 65-74 years. However, in the latter age group prevalence declined at the end of the period (Fig. 4). Among the oldest women and the oldest men ( $\geq 75$ years) prevalence decreased over the whole study period.

For the last 15 years of the study period, the mean age of onset for men decreased from 64 to 59 years. For women the mean age of onset was 65 years throughout the study period.

\section{Discussion}

The prevalence of diabetes mellitus in the municipality of Laxå is high, but there seems to have been no rise during recent years. The incidence rate was relatively stable over the 30-year period, despite some fluctuation, with the highest rate during the first screening period 1983-1987.

A changing prevalence of diabetes may theoretically be due to new diagnostic criteria, change in the ratio of diagnosed to undiagnosed cases, the efficiency of the casefinding procedure, demographic changes, the age at onset of the disease, changes in the survival rate and a true change in incidence rates.

Changing diagnostic criteria In this study the diagnostic criteria were changed three times according to the WHO definition [13-15], which should have led to more cases being detected. This was certainly the case in 1980 when the long-awaited new diagnostic criteria established a solid base for the diagnosis of diabetes. The change in criteria in 1985 was too modest to make any impression on incidence and prevalence rates. More unexpected, however, was the fact that the lowering in 1999 of the diagnostic criteria for 
Fig. 3 Prevalence of diabetes for women expressed as agestandardised number of prevalent cases per 1,000 residents per year in the community of Laxå. Blue line, women 0 24 years; red line, women 25 34 years; yellow line, women 35-44 years; green line, women 45-54 years; purple line, women 55-64 years; orange line, women 65-74 years; brown line, women 75 years and older; black line, all women

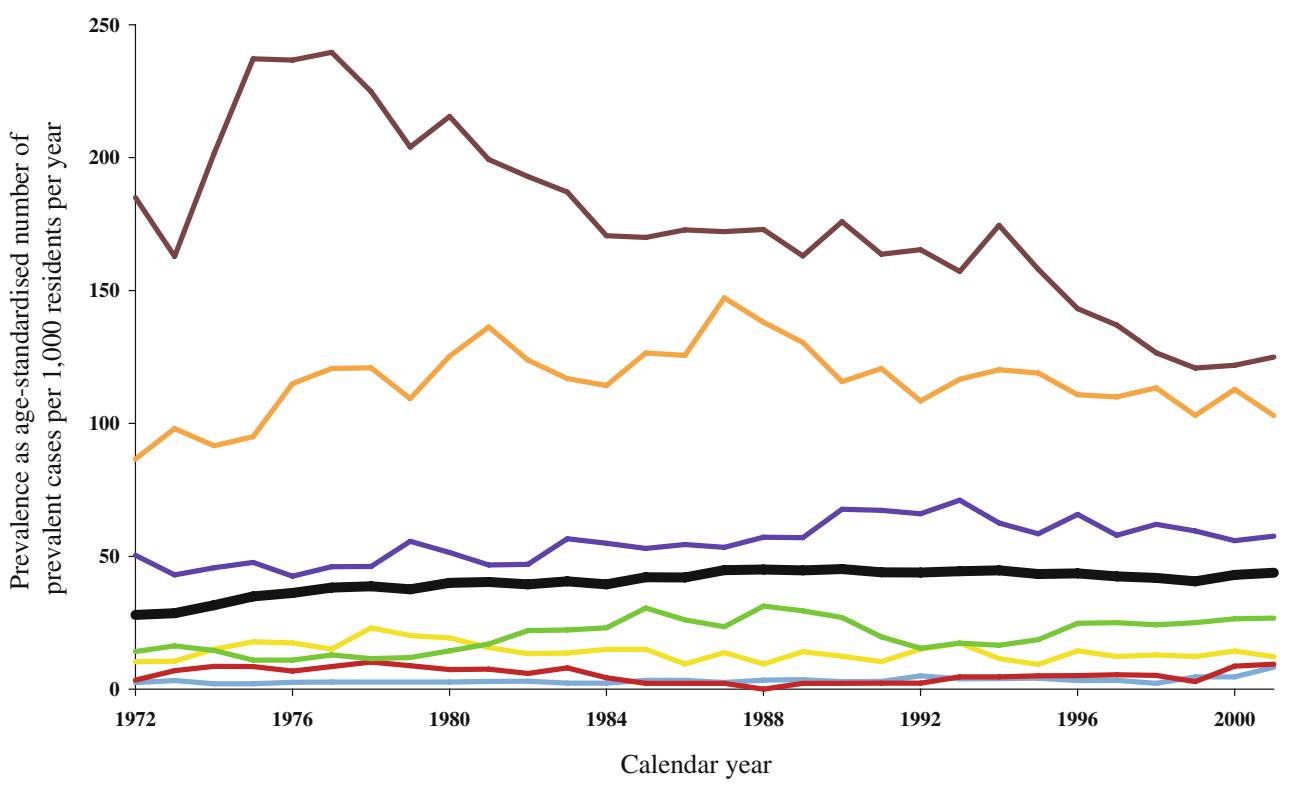

fasting blood glucose from 6.7 to $6.1 \mathrm{mmol} / \mathrm{l}$ did not lead to an increase in incidence and prevalence rates. We believe that this is explained by the liberal use of OGTTs among subjects with what is now known as impaired fasting glucose during 15 years of case-finding, which had already unmasked individuals with diabetes on the basis of 2-h OGTT glucose values.

Ratio of diagnosed to undiagnosed diabetic subjects During the 30-year period Laxå residents had limited access to medical services other than those provided by the local PHCC. As a result of this, over $90 \%$ of the inhabitants in the 35- to 79-year age group had permanent contact with the local PHCC. At this unit, only a few physicians and a diabetes nurse using uniform diagnostic criteria were involved in the care of diabetic patients. A diabetes registry spanning the whole period and complete medical records for all diabetic patients were available at the PHCC.

From 1983, high-risk individuals were screened for diabetes almost yearly and other Laxå residents every 5 years. These factors all favoured optimal conditions for diagnosing patients with diabetes, both before, and even more so during the opportunistic case-finding periods. Compared with a situation with regular OGTT screening in the community, the prevalence rates are probably somewhat underestimated, and particularly during the period 1973-1982, when few OGTTs were done, due to unclear diagnostic criteria at that time. For the period from
Fig. 4 Prevalence of diabetes for men expressed as agestandardised number of prevalent cases per 1,000 residents per year in the community of Laxå. Blue line, men 0-24 years; red line, men 25-34 years; yellow line, men 35-44 years; green line, men 45-54 years; purple line, men 55-64 years; orange line, men 65-74 years; brown line, men 75 years and older; black line, all men

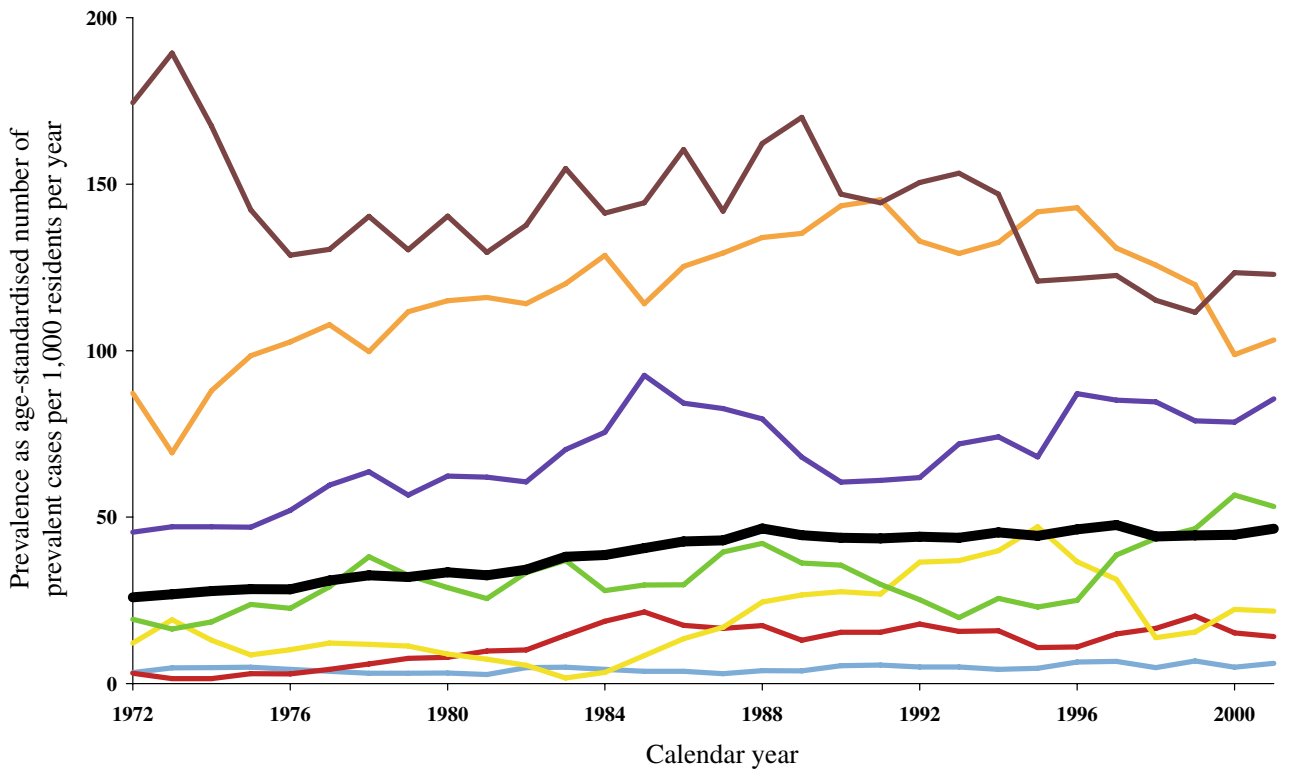


1983 to 2001 the case-finding activities were adjusted to the prevailing diagnostic criteria, but were otherwise uniform over time, with OGTTs performed liberally when fasting glucose levels proved inconclusive. Therefore, it is unlikely that the absence of repeated OGTT screening activities more than marginally influenced prevalence time trends. However, age at diagnosis of diabetes may have been somewhat higher than if OGTTs had been the primary screening method.

Efficiency of the opportunistic case-finding procedure The efficiency of the case-finding procedure is closely tied to the ratio of diagnosed to undiagnosed diabetes cases. When the first case-finding period began in 1983, this was the start of a more intense search for undiagnosed cases of type 2 diabetes. It was much more efficient than the irregular testing done before, leading to prompt increases in both incidence and prevalence rates. Over time, the glucose value that represented a positive screening test was lowered in response to new diagnostic criteria, thereby maintaining an equivalent case-finding efficiency.

Demographic changes During the study period, 118 diabetic patients moved out of and 48 moved into Laxå, changes that are similar to migration among the nondiabetic population. The Laxå population has grown older from the mid 1980s onwards, which may explain the rise in the crude prevalence rate seen during this period. This development, however, should not influence age-standardised rates.

Age at onset of diabetes Age at actual onset of diabetes is very difficult to determine, in contrast to age at diagnosis. Together with survival, age at onset or diagnosis determines disease duration. At the end of the study period, we saw a shift towards younger age at diagnosis, at least in men. An earlier age at diagnosis should be expected during the study period due to the more intense search for people with diabetes, but our findings do not rule out the possibility of a real reduction in age at onset of the disease.

Changes in survival rate In recent years, the mortality rate for cardiovascular disease in Sweden has declined and the population lives longer, but it is unclear whether this holds true for the diabetic population. In a forthcoming paper, we aim to analyse whether the survival of subjects with type 2 diabetes has improved in the same way as that for nondiabetic subjects during the 30-year period. For the time being we only know that 582 diabetic patients died during the study period.

True change in incidence rates We found no obvious change in the true incidence rate of type 2 diabetes during the study period. The fluctuations in incidence rate that were seen during the first 15 years are probably artefacts and can be explained by fluctuating levels of effort to find diabetic persons during these years. During the period from 1987 to 2001, when the case-finding activities were conducted uniformly, it should have been possible to detect any true change in incidence rate. However, no such change could be seen.

A common opinion among diabetologists is that increasing levels of overweight and a more sedentary lifestyle in a population should result in more persons getting type 2 diabetes. We have no reason to believe that our Laxå residents are resistant to obesity or to a physically inactive daily life. Therefore, an increase in incidence rate would have been expected, although no such change was seen. Perhaps our study period, although long, was too short to unveil any changes. Another reason for the absence of a rise in incidence could be that our Europid population is not as vulnerable to diabetes as some other populations. If this holds true, more years of exposure to obesity and a sedentary life might be necessary before diabetes develops. It is also possible that the population consists of two groups, one more vulnerable, and the other relatively resistant to contracting diabetes. Adverse lifestyle factors might then afflict the vulnerable group by lowering the age at onset, but still leave the other group unaffected. In such a scenario, the incidence rate would be fairly stable. The lesson learned is perhaps that each population deserves its own study. Therefore we really cannot say that there is an epidemic of diabetes, at least not in Laxå.

Conclusions Of all the factors contributing to the prevalence of diabetes, we conclude, after several years of casefinding, that we have had a stable incidence rate over a long period of time. We observed an earlier onset of the disease, at least for men. We do not yet know if the diabetic population has increased survival in line with that seen in the general population. In our study, it seems that prevalence has maintained a fairly constant level over a relatively long period. However, the explanation is unclear, and as discussed recently [16] multiple factors influence the overall prevalence of diabetes.

Acknowledgements This study was supported by grants from the Primary Healthcare Research Unit, Örebro, the Örebro County Council Research Unit, the Family Medicine Research Centre, Örebro University and Uppsala University (all in Sweden).

Duality of interest D. K. G. Andersson and K. Svärdsudd have no relevant dualities of interest to declare.

S. P. O. Jansson has received honoraria for speaking engagements from GlaxoSmithKline, Novo Nordisk and Sanofi-Aventis. 


\section{References}

1. World Health Organization (2006) The prevention of diabetes and its complications. Available from http://www.who.int/diabetes/ preventionflyer/en/ (last accessed 8 September 2006)

2. Wild S, Roglic G, Green A, Sicree R, King H (2004) Global prevalence of diabetes: estimates for the year 2000 and projections for 2030. Diabetes Care 27:1047-1053

3. Wareham NJ, Forouhi NG (2005) Is there really an epidemic of diabetes? Diabetologia 48:1454-1455

4. Andersson DK, Svardsudd K, Tibblin G (1991) Prevalence and incidence of diabetes in a Swedish community 1972-1987. Diabet Med 8:428-434

5. Berger B, Stenstrom G, Chang YF, Sundkvist G (1998) The prevalence of diabetes in a Swedish population of 280,411 inhabitants. A report from the Skaraborg Diabetes Registry. Diabetes Care 21:546-548

6. Glumer C, Jorgensen T, Borch-Johnsen K (2003) Prevalences of diabetes and impaired glucose regulation in a Danish population: the Inter99 study. Diabetes Care 26:2335-2340

7. Yliharsila H, Lindstrom J, Eriksson JG et al (2005) Prevalence of diabetes and impaired glucose regulation in 45- to 64-year-old individuals in three areas of Finland. Diabet Med 22:88-91

8. Drivsholm T, Ibsen H, Schroll M, Davidsen M, Borch-Johnsen K (2001) Increasing prevalence of diabetes mellitus and impaired glucose tolerance among 60-year-old Danes. Diabet Med 18:126-132
9. Midthjell K, Kruger O, Holmen J et al (1999) Rapid changes in the prevalence of obesity and known diabetes in an adult Norwegian population. The Nord-Trondelag Health Surveys: 1984-1986 and 1995-1997. Diabetes Care 22:1813-1820

10. Eliasson M, Lindahl B, Lundberg V, Stegmayr B (2002) No increase in the prevalence of known diabetes between 1986 and 1999 in subjects 25-64 years of age in northern Sweden. Diabet Med 19:874-880

11. Eliasson M, Boström G (2006) Chapter 5.2: major public health problems - diabetes. Scand J Public Health Suppl 67:59-68

12. Andersson DK, Lundblad E, Svardsudd K (1993) A model for early diagnosis of type 2 diabetes mellitus in primary health care. Diabet Med 10:167-173

13. World Health Organization (1980) WHO expert committee on diabetes mellitus: second report. World Health Organization Technical Report Series no. 646:1-80

14. World Health Organization (1985) Diabetes mellitus. Report of a WHO study group. World Health Organization Technical Report Series no. 727:1-113

15. World Health Organization (1999) Definition, diagnosis and classification of diabetes mellitus and its complications: report of a WHO consultation. Part 1: Diagnosis and classification of diabetes mellitus. WHO, Department of Noncommunicable Disease Surveillance, Geneva

16. Colagiuri S, Borch-Johnsen K, Glumer C, Vistisen D (2005) There really is an epidemic of type 2 diabetes. Diabetologia 48:1459-1463 